\title{
Experiences Porting the SU3_Bench Microbenchmark to the Intel Arria 10 and Xilinx Alveo U280 FPGAs
}

\author{
Douglas Doerfler \\ Farzad Fatollahi-Fard \\ Colin MacLean \\ Tan Nguyen \\ Samuel Williams \\ Nicholas J. Wright \\ dwdoerf@lbl.gov \\ ffard@lbl.gov \\ colinmacLean@lbl.gov \\ tannguyen@lbl.gov \\ swwilliams@lbl.gov \\ njwright@lbl.gov \\ Lawrence Berkeley National Laboratory \\ Berkeley, CA, USA
}

\author{
Marco Siracusa \\ DEIB, Politecnico di Milano \\ Milano, Italia \\ marco.siracusa@mail.polimi.it
}

\begin{abstract}
In this study we investigate the implications of porting a common computational kernel used in high performance computing, which has been optimized for efficient execution on general purpose graphics processing units (GPUs), to a field programmable gate array (FPGA). In particular, we use a benchmark based on a matrix-matrix multiply kernel commonly used in lattice quantum chromodynamics applications. The microbenchmark is based on the OpenCL programming language. We evaluate the performance, and portability, aspects associated for two FPGAs, the Intel Arria 10 and the Xilinx Alveo U280. The purpose of the study is not to compare the two FPGAs, but to evaluate their respective OpenCL toolchains and to evaluate the level of effort needed to port a GPU optimized code to a FPGA, and the effectiveness of the respective toolchains. We did find the toolchains to be relatively easy to use, and it was possible to get correctness with little effort, but there was significant effort needed to get relatively good performance. We found that FPGAs perform best when using single work item kernels, as opposed to the nominal multiple work item NDRange kernel used for CPUs and GPUs. In addition, other source code changes were necessary, and in particular the lack of a local cache in FPGA architectures can require a significant rewrite of the code. The performance achieved with the Intel Arria 10 was $47.6 \%$ of its maximum sustained bandwidth, while the Xilinx Alveo U280 achieved $35.2 \%$. GPU architectures have been shown to demonstrate $75 \%$ to $90 \%$ architectural efficiencies.
\end{abstract}

This work is licensed under a Creative Commons Attribution International 4.0 License. IWOCL'21, April 27-29, 2021, Munich, Germany

(C) 2021 Copyright held by the owner/author(s).

ACM ISBN 978-1-4503-9033-0/21/04.

https://doi.org/10.1145/3456669.3456671

\section{KEYWORDS}

FPGA, OpenCL, Portability, Performance, Lattice Quantum Chromodynamics

\section{ACM Reference Format:}

Douglas Doerfler, Farzad Fatollahi-Fard, Colin MacLean, Tan Nguyen, Samuel Williams, Nicholas J. Wright, and Marco Siracusa. 2021. Experiences Porting the SU3_Bench Microbenchmark to the Intel Arria 10 and Xilinx Alveo U280 FPGAs. In International Workshop on OpenCL (IWOCL'21), April 2729, 2021, Munich, Germany. ACM, New York, NY, USA, 9 pages. https: //doi.org/10.1145/3456669.3456671

\section{INTRODUCTION}

Matrix-matrix (and matrix-vector) SU(3) operations are a fundamental building block of Lattice Quantum Chromodynamics (QCD) applications $[1,3]$. The SU3_Bench microbenchmark was developed to explore different programming methodologies, and the $\mathrm{SU}(3)$ matrix-matrix operation is used as the computational kernel [12]. Although SU3_Bench was primarily developed to explore acceleration using general purpose graphics processing units (GPU), in this study we extend the OpenCL version of the microbenchmark to target FPGAs as an accelerator, and explore the maturity and performance potential of the Intel and Xilinx Open Computing Language (OpenCL) compiler environments.

We limit ourselves to OpenCL, as opposed to the more common high-level synthesis (HLS) toolchain used by most FPGA developers, as we deem it to be the most suitable portability layer for high performance computing (HPC) application developers and it allows us to compare kernel development across multiple FPGA architectures using a standard language. Although the SU3_Bench microbenchmark is portable across GPUs, and traditional multicore microprocessors based on central processing units (CPU), we limit this study to a comparison of two FPGA based accelerator cards.

In order to best illustrate the the numerous source code choices in implementing the SU3_Bench kernel, source code listings are 
provided in Appendix A. We examine two types of OpenCL kernel implementations, NDRange and single work item (SWI) kernels.

Table 1: Listing Descriptions (see Appendix A)

\begin{tabular}{|l|c|}
\hline Listing & Description \\
\hline 1 & Nominal NDRange \\
\hline 2 & NDRange with unroll \\
\hline 3 & NDRange with 16 workers \\
\hline 4 & Nominal SWI \\
\hline 5 & SWI with unroll \\
\hline 6 & SWI with scratch pad \\
\hline 7 & SWI with scratch pad and compute integrated \\
\hline
\end{tabular}

A NDRange kernel is nominally the method used when writing parallel OpenCL kernels with multiple work items, and in this case a call to the OpenCL function get_global_id() is used to determine the work item index, as illustrated in Listing 1. A SWI kernel is essentially a sequential implementation in which all data is processed by a single work item, Listing 4 . For a complete description of OpenCL programming modes, refer to [9].

The unique contribution provided by this study is the evaluation of two different vendor OpenCL compiler toolchains on two respective devices, and the portability considerations in moving between the two. It is also a representative case study for issues an application developer will face when attempting to target a FPGA device as an accelerator using OpenCL.

\section{TARGET FPGA ARCHITECTURES}

In this study, two FPGA boards were used for the analysis. The basic architecture for both devices is shown in Figure 1, and the specifications of interest are in Table 2. The devices were both installed into the same host system, which contains dual, 8-core Intel(R) Xeon(R) CPU E5-2650 v2 CPUs clocked at 2.60GHz. The hardware details of the devices can be found in the respective data sheets $[7,14]$.

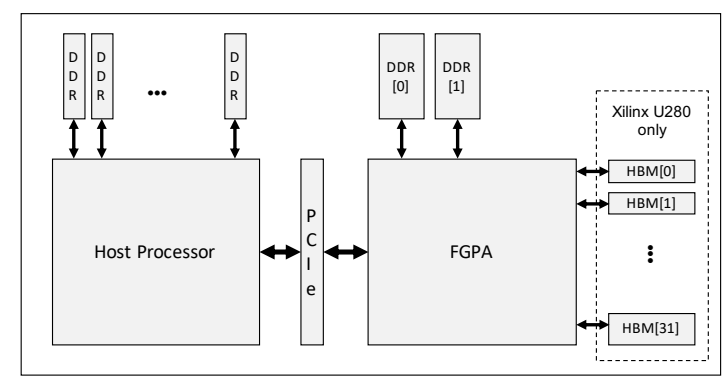

Figure 1: Test Platform

This study is not a comparison of one device to another, and it would be unfair as the two devices have dramatically different design points and capabilities.

The SU3_Bench benchmark is inherently memory bound, and hence absolute performance is dependent on the bandwidth characteristics of the memory subsystem used for OpenCL global memory.
Table 2: FGPA Specifications

\begin{tabular}{|l|l|l|}
\hline & $\begin{array}{l}\text { Intel Arria 10 } \\
\text { GX }\end{array}$ & $\begin{array}{l}\text { Xilinx Alveo } \\
\text { U280 }\end{array}$ \\
\hline \# DDR Channels & 2 & 2 \\
\hline DDR Capacity (GB) & 8 & 32 \\
\hline DDR Peak BW (GB/s) & 34.1 & 38.4 \\
\hline \# HBM Channels & N/A & 32 \\
\hline HBM Peak BW (GB/s) & N/A & $460(14.1 / \mathrm{ch})$. \\
\hline Toolchain & Quartus Prime & $\begin{array}{l}\text { Vitis Unified } \\
\text { Software }\end{array}$ \\
\hline Compiler Version & Version 17.1.1 & v2019.2.1 \\
\hline
\end{tabular}

For the Intel Arria 10, this is a DDR based memory with two DDR channels configured to interleave pages across the two channels. For the Xilinx Alveo U280, it contains DDR and HBM memory subsystems. For this study, only the higher performing HBM was analyzed.

The Xilinx Alveo U280 has two channels of DDR, and 32 "pseudo channels" of HBM. For this study, we only benchmark the performance of the higher bandwidth HBM. The HBM of this device is not designed to be operated as a single, monolithic unit, and is instead best taken advantage of as individual channels. Xilinx recommends not using a single HBM channel for read/write operations as there is a bus turnaround overhead for the controller to switch between the two modes [14, p. 14]. It is preferred to have channels dedicated for reads or writes. For this analysis, channel HBM[0] is used for read-only variables, and HBM[1] is used for write-only variables and the remaining 30 channels are unused at this time. One of our design constraints for this study is to maximum the performance using these two channels, and then replicate the design across the remaining channels to fully utilize the HBM, and/or the device, but this is reserved for a future study.

In the following analysis, we measure absolute performance and compare that against the maximum sustained performance for each FPGA. For both devices, a roofline analysis was performed using the Empirical Roofline Toolkit (ERT) [13]. In addition, Xilinx provides performance information for the Alveo U280 under ideal conditions [14]. For this analysis, only 2 of the 32 HBM pseudo channels were used with one channel dedicated for read operations and another dedicated for write operations. Since these operations were sequential in our Xilinx design, the expected performance is that of a single channel. These maximum bandwidths are captured in Table 3.

Table 3: Peak \& Sustained Bandwidths

\begin{tabular}{|l|c|c|}
\hline & Peak GB/s & Sustained GB/s \\
\hline $\begin{array}{l}\text { Intel Arria 10 (interleaved } \\
\text { DDR) }\end{array}$ & 34.1 & 25.0 \\
\hline $\begin{array}{l}\text { Xilinx Alveo U280 (per } \\
\text { HBM channel) }\end{array}$ & 14.4 & 13.1 \\
\hline
\end{tabular}

As a point of reference, similar studies of SU3_Bench on GPU architectures have demonstrated performances of $75 \%$ to $90 \%$ of 
their respective architectural rooflines [4]. One goal of this study is to achieve comparable performance on FPGA architectures.

\section{THE SU3 BENCH MICROBENCHMARK}

The nominal SU3_Bench NDRange kernel is shown in Listing 1, Appendix A. In this implementation each thread (OpenCL work item) operates on a single lattice site, which consists of doing an $\mathrm{SU}(3)$ matrix-matrix multiply for each of the four (4) dimensions. Each SU(3) matrix is a $3 \times 3$ matrix of complex, floating-point values and hence a matrix-matrix multiply consists of nine (9) $3 \times 1$ vector dot product operations. Taking into account the four dimensions, this is a total of 36 dot products for each site.

The SU3_Bench kernel takes as input parameters the variable $a$, the source lattice, $b$, a constant four dimensional SU(3) matrix that operates on $a$, and $c$, the resultant lattice. Since $b$ is constant, and of relatively small size, it is expected to reside in L1 cache and hence the memory traffic consists of reading $a$ and writing $c$. If a cache isn't available, then the kernel must explicitly copy $b$ to kernel local memory.

For this study we use complex, single-precision floating point operations. Although double-precision is most often used in HPC applications, the use of mixed precision algorithms is common in Lattice QCD applications where the bulk of the computation is done in single-precision while the higher level accumulations are performed in double-precision [3]. FPGAs typically have single-precision fixed function logic in their digital signal processing blocks, including the devices used in this study. The higher level accumulations could be done by a double-precision unit implemented in FPGA logic blocks, or it could be done by the host processor, but that is beyond the scope of this analysis.

A site is of size 320 bytes, 288 bytes for the su3_matrix elements and the remaining bytes for metadata and padding to a 64 byte boundary (see Appendix ??). Thus for each site, 320 bytes of data are read and 320 bytes of data are written for a total of 640 bytes. The SU(3) matrix-matrix multiply takes 864 floating-point operations (FLOPs). Thus, the arithmetic intensity (FLOPs to Bytes ratio) is calculated to be 1.35 . For most architectures, this is a relatively low arithmetic intensity and hence the SU3_Bench calculation tends to be limited by the realized bandwidth of the memory subsystem architecture [4].

SU3_bench measures the time to enqueue the kernel and perform the matrix-matrix multiply calculation, ending in the completion of the kernel and return of control to the host program. It does not include the time to move the data to and from the device over the PCIe bus from host global memory. Given the known theoretical data movement and FLOP count, the host program calculates and reports rates in GigaFLOPs/second and GigaBytes/second. For the Arria 10 , the size of the lattice is $L=32$ for a total of $32^{4}=1,048,576$ sites and a total footprint of 640 mebibytes for the arrays $a$ and $c$. Array $b$ is relatively small and is expected to fit in a local cache or a scratch pad memory. For the Alveo U280, the size of the lattice was reduced to $\mathrm{L}=16,40$ mebibytes in aggregate across the arrays, in order to fit in a single bank of HBM which has a capacity of $256 \mathrm{MiB}$. Since SU3_Bench is inherently memory bandwidth bound, the size of the lattice is not critical as long as it is sufficiently larger than any local cache, which is not an issue for either FPGA.

\section{INTEL ARRIA 10 FPGA}

\subsection{Results \& Analysis}

The best performance was obtained with Listings 2 and 5 for NDRange and SWI kernels respectively. These kernels simply add a single \#pragma unroll directive above the outer $j$ loop. Results are shown in Table 4. The SWI kernel gives the best absolute performance, $11.9 \mathrm{~GB} / \mathrm{s}$, a $3.0 x$ speedup over the NDRange kernel's $3.95 \mathrm{~GB} / \mathrm{s}$.

Note that other Intel Quartus optimizations were explored including \#pragma loop_coalesce and

__attribute_((num_simd_work_items $(N)))$. These design options demonstrated slightly worse performance than unrolling and hence further exploration was deemed unfruitful.

Table 4: Arria 10 Performance

\begin{tabular}{|l|c|c|c|c|}
\hline & Type & GF/s & GB/s & $\%$ Max. \\
\hline Listing 2 & NDRange & 5.33 & 3.95 & $15.8 \%$ \\
\hline Listing 2 w/4 CUs & NDRange & 4.68 & 3.47 & $13.9 \%$ \\
\hline Listing 5 & SWI & 16.1 & 11.9 & $47.6 \%$ \\
\hline $\begin{array}{l}\text { Listing 5 no inter- } \\
\text { leave }\end{array}$ & SWI & 9.38 & 6.95 & $27.8 \%$ \\
\hline $\begin{array}{l}\text { Listing 5 no inter- } \\
\text { leave, 2 channels }\end{array}$ & SWI & 18.0 & 13.3 & $53.2 \%$ \\
\hline \multicolumn{5}{|c|}{ Without Computation } \\
\hline Listing 2 NDRange & 310.0 & 229.6 & \\
\hline Listing 5 & SWI & 7,508 & 5,562 & \\
\hline
\end{tabular}

Nominally, the two DDR channels are configured to interleave pages. To understand the overhead of interleaving, a version of the SWI kernel was created in which the DDR channels were not interleaved. In this configuration, one-half the full memory address range is mapped to the first channel and one-half is mapped to the second channel. If the size of the problem is controlled to reside entirely in the first channel, the performance was measured at $6.95 \mathrm{~GB} / \mathrm{s}$. Nominally we'd expect a slowdown of $2 x$, but the observed slowdown is $1.71 x$. Increasing the problem size to fill the entire address range, both channels are active with the array $a$ residing in Channel 0 and array $c$ residing in Channel 1 . This results in a performance of $13.3 \mathrm{~GB} / \mathrm{s}$, a speedup of $1.12 x$ over the interleaved design. This simple test shows that there is a significant gain in performance when using the memory in a non-interleaved mode, but the use case of fully occupying memory capacity is not very practical, and for decreasing problem size the advantage diminishes to the performance of a single channel.

The advantage of using a NDRange kernel design is it eases the use of multiple compute units (CU). With a SWI kernel, a single $\mathrm{CU}$ processes the entire problem range. It is still possible to create multiple CUs with a SWI kernel, but this also requires the host program to partition the problem and call separate kernels. From a portability perspective this is not desirable, a NDRange approach is preferable. Unfortunately, the performance of the NDRange design in the FPGA is $3 x$ slower.

To better understand this overhead, kernels were created in which no compute takes place. Since the FPGA kernel code is compiled separately from the host code, the kernel compiler may optimize out the nested loops, but it still must go through the device 
queue and get scheduled (albeit to do nothing). This essentially sets a limit on performance where the limitation is the runtime overhead. The performance for the "no compute" kernels are also shown in Table 4, for both a NDRange kernel and a SWI kernel. Performance is $229.6 \mathrm{~GB} / \mathrm{s}$ and $5,562 \mathrm{~GB} / \mathrm{s}$ respectively. This demonstrates the extra overhead of the runtime associated with an NDRange kernel.

The above analysis only used a single CU for the NDRange kernel. The number of work items per CU was configured to be four using_attribute_((reqd_work_group_size $(4,1,1)))$ for the kernel. However, increasing the number of work items per CU doesn't result in additional hardware logic being used and the performance remains the same, indicating the workers are scheduled sequentially.

To see if a multi-CU design would improve performance, a configuration was created with four CUs using the additional

__attribute_((num_compute_units(4))). Hence there are 4 CUs with 4 workers each for a total of 16 workers. This design did use more hardware logic, but still comfortably resided within the Arria 10 resources. Again, the number of work items per CU was configured to be four. Hence there are 4 CUs with 4 workers each for a total of 16 workers. The $4 \mathrm{CU}$ design actually demonstrated reduced performance, a slowdown of $1.14 x$, despite using additional hardware resources. Again demonstrating an overhead in the NDRange kernel runtime.

A future study may look into these NDRange runtime issues. But in short, this is a major limitation of the Intel FPGA runtime, as it is highly desirable that NDRange kernels perform well to ease the transition for GPU based codes.

\subsection{Portability Considerations}

4.2.1 Using Block RAM as a cache. The Intel Quartus toolchain generates a cache out of block RAM to accompany load store units (LSU) for the global memory subsystem, the size of the cache can be specified at compile time, but for these kernels it chose to use a 64 Kibibit $(8 \mathrm{KiB})$ cache for each LSU generated. Because of this, it is not necessary to explicitly copy $b$ to a local variable and kernel modifications are limited to explicit pragma directives for FPGA optimization.

4.2.2 Use SWI Kernels. Intel's Best Practices Guide for programming FPGAs recommends that SWI kernels be constructed, as opposed to OpenCL NDRange kernels [8, Sec. 1.3]. The compiler performs more aggressive optimizations, such as pipelining, with SWI kernels. Although it is not stated in the Best Practices Guide, it has been observed that when compiling NDRange kernels it uses less aggressive optimizations in order to reduce resource usage and hence allow the kernel to be replicated into multiple work items. Explicitly forcing pipelining cannot be achieved because the compiler doesn't provide a directive to suggest pipelining a loop, only one to not use a pipeline when the compiler wants to do one implicitly.

There were many trials (and tribulations) using different combinations of pragma directives, but the best performance was obtained with a single \#pragma unroll directive above loop $j$ in Listings 2 and 5 for the NDRange and SWI kernels respectively.

Performance of the NDRange was measured to be $3.95 \mathrm{~GB} / \mathrm{s}$ and SWI kernels was measured at $11.9 \mathrm{~GB} / \mathrm{s}$. Referring to Table 3, the respective kernel performance is $15.8 \%$ and $47.6 \%$ of the maximum sustained bandwidth of the device. The reason for the performance difference was verified by looking at the optimizations performed, as reported by the compiler reports. The higher optimization of the SWI kernel is due to the explicit unrolling of loop $j$, implicit pipelining of loop $k$ and the implicit unrolling of loop $\mathrm{m}$. For the NDRange kernel, loop $k$ is not pipelined, as the compiler disables pipelining for an NDRange kernel, as discussed above.

\section{XILINX ALVEO U280 FPGA}

\subsection{Results \& Analysis}

The Alveo U280 HBM architecture consists of 32 pseudo channels. For this study, the kernels were configured to only use two channels, HBM[0] and HBM[1]. The read-only $a$ and $b$ variables were mapped to $\operatorname{HBM}[0]$, while the write-only $c$ variable was mapped to HBM[1]. This was done to avoid the extra overhead of switching between reads and writes on a single channel and hence maximizing bandwidth. Note that since the kernel design does not use a dataflow architecture between the Read $\rightarrow$ Compute $\rightarrow$ Write stages of the kernels, the sequential nature of the design means there is no overlap between reads and writes and hence the maximum bandwidth achieved is that of a single channel, Table 3.

Performance results are shown in Table 5.

Table 5: Alveo U280 Performance

\begin{tabular}{|l|c|c|c|c|}
\hline & Type & GF/s & GB/s & $\%$ Max \\
\hline Listing 3 & NDRange & 0.401 & 0.297 & $2.27 \%$ \\
\hline Listing 3 w/4 CUs & NDRange & 0.613 & 0.454 & $3.47 \%$ \\
\hline Listing 4 & SWI & 0.225 & 0.167 & $1.27 \%$ \\
\hline Listing 6 & SWI & 6.23 & 4.61 & $35.2 \%$ \\
\hline Listing 7 & SWI & 1.66 & 1.23 & $9.39 \%$ \\
\hline
\end{tabular}

The best performance, $4.61 \mathrm{~GB} / \mathrm{s}$, was obtained with the SWI kernel of Listing 6, which is a modified version of the nominal SWI kernel of Listing 4. Listing 6 uses additional scratch pad logic to account for the lack of a local cache in the Xilinx device, which is further described in Section 5.2. To demonstrate the importance of using scratch pads, the nominal SWI kernel of Listing 4, was benchmarked. Relative to Listing 6, performance is over $27 x$ slower.

The NDRange kernel for the Alveo U280 is shown in Listing 3. This kernel is a modified version of Listing 1 in which local arrays are used for scratch pads and the number of workers is set to the size of the scratch pads, in this case 16 to match the scratch pad size used in the SWI kernel. But the performance of Listing 3 is $15.5 x$ slower than Listing 6, $0.297 \mathrm{~GB} / \mathrm{s}$ versus $4.61 \mathrm{~GB} / \mathrm{s}$. Referring to Table 3, the respective kernel performance is $2.27 \%$ and $35.2 \%$ of the maximum sustained bandwidth of the device. As with the Intel Arria 10, the Xilinx Alveo U280 NDRange performance is significantly lower than that achieved with an SWI kernel.

In Listing 7 , the $j, k$ and $l$ loops were combined for reading $a \_b u f$ and the computation, an elegant modification of Listing 6 from a programming perspective, but at the result of decreased performance because the compiler doesn't schedule the burst reads of $a$. 
The Xilinx Vitis toolchain provides for the collection of profile data in the hardware design. With this enabled, several metrics are collected, but for this analysis it is interesting to look at the time spent stalled on global memory transfers. In the performance analysis above, the use of local memory scratch pads and enabling burst memory transfers from global memory is key to performance. However, this also divides the calculation into three sequential stages: Read $\rightarrow$ Compute $\rightarrow$ Write, and hence adds extra cycles to the calculation. Profile data are shown in Table 6.

Table 6: Alveo U280 Stalls to Global Memory

\begin{tabular}{|l|l|l|l|l|l|}
\hline & Type & $\begin{array}{l}\text { Runtime } \\
(\mathrm{ms})\end{array}$ & $\begin{array}{l}\text { Active } \\
(\mathrm{ms})\end{array}$ & $\begin{array}{l}\text { Stalls } \\
(\mathrm{ms})\end{array}$ & $\begin{array}{l}\text { Stalls } \\
(\%)\end{array}$ \\
\hline Listing 3 & NDRange & 165.27 & 163.24 & 2.03 & $1.23 \%$ \\
\hline $\begin{array}{l}\text { Listing 3 } \\
\text { w/4 CUs }\end{array}$ & NDRange & 93.17 & 91.10 & 2.08 & $2.23 \%$ \\
\hline Listing 4 & SWI & 251.91 & 3.41 & 248.51 & $98.6 \%$ \\
\hline Listing 6 & SWI & 9.20 & 5.78 & 3.42 & $37.2 \%$ \\
\hline Listing 7 & SWI & 34.84 & 10.33 & 24.52 & $70.4 \%$ \\
\hline
\end{tabular}

The number of active cycles doing computation is lowest for Listing 4 without local memory scratch pads, $3.41 \mathrm{~ms}$, but the utilization of the memory subsystem is very inefficient and there is a large number of stalls accessing global memory, contributing to over $98 \%$ of the total runtime. In Listing 6 with local memory scratch pads, runtime decreases by a factor of $27 x$ to $9.20 \mathrm{~ms}$, but the time for the calculation increases to $5.78 \mathrm{~ms}$ due to the time taken to copy the global memory to/from local scratch pads, but this is much more efficient overall as the time spent stalled on global memory decreases by a factor of $73 x$. We also see that Listing 7 (where the read of $a$ and the computation is combined into a single loop nest) is less efficient than Listing 6, both in the time taken to do the calculation and the time due to stalls to global memory.

\subsection{Portability Considerations}

5.2.1 Unrolling vs. Pipelines. For the Xilinx kernels, the compiler does a good job of automatically unrolling loops when it deems it appropriate. But it does require explicit pipeline directives. This is the opposite of the behavior of the Intel toolchain, which requires explicit unroll directives and implicitly decides when and when not to pipeline. In fact the Intel compiler lacks a pipeline directive (but does provide one to tell the compiler not to pipeline).

5.2.2 Creating local memory scratch pads. The Xilinx Vitis toolchain [15] does not generate a cache for its load store units, and hence local memory scratch pads need to be used and the kernel must explicitly copy data from global memory to local memory. The NDRange kernel demonstrating the use of local memory scratch pads is shown in Listing 3, and the SWI kernel in Listing 6.

The user is dependent on the compiler detecting streaming transfers and translating them into 512 bit burst transfers which maximize memory controller bandwidth. For example, in Listing 7 the calculation is moved into the same nested loop structure as the read of the $a \_b u f$ scratch pad. In this case, the compiler doesn't generate 512 bit burst transfers and instead sequential 64 bit transfers are used. This dramatically decreases read bandwidth and causes stalls in the computational pipeline resulting in $3.8 \mathrm{x}$ lower performance when compared to Listing 6, despite this being a more intuitive kernel from a programmer perspective.

5.2.3 Ensuring 512 bit burst transfers. Xilinx documents that the peak HBM bandwidth occurs when transfers 512 bits (64 bytes) or larger with sequential addressing [14, p. 14]. If this condition is met, and the compiler detects it can perform burst transfers, the memory controller can achieve $91 \%$ of peak HBM bandwidth, $\sim 13.1 \mathrm{~GB} / \mathrm{s}$. A 32 byte transfer is significantly less efficient, $4.5 \mathrm{~GB} / \mathrm{s}$. Hence it's critical to ensure 512 bit transfers are scheduled. For Listing 6, the compiler does indeed schedule 512 bit transfers, however achieved performance is still only $35.2 \%$ of the maximum sustained bandwidth.

Another key element is the use of the site structure for $a$ and $c$. A site is of size 320 bytes, 288 bytes for the su3_matrix elements and the remaining bytes for metadata and padding to a 64 byte boundary. The compiler recognizes this as five 512 bit transfers and hence schedules a burst transfer of length five. However, for reading in $b \_b u f$, the compiler schedules 64 bit transfers, which is assumed because 288 bytes is not an integer multiple of 64 bytes. The author did a modified version of the kernel in which $a$ and $c$ where also su3_matrix* parameters, and the compiler again scheduled 64 bit transfers.

It is critical that the input parameters to a kernel are wrapped in appropriate structures that allow the compiler to recognize the extent of the data transfer and enable it to schedule the most efficient transfers.

5.2.4 Host program source code changes. The Xilinx port also required a change to the host program. In allocating OpenCL Buffer objects, the Xilinx runtime requires that the the flag

$C L \_M E M \_U S E \_H O S T \_P T R$ be set. It's not clear why the Xilinx runtime wants it set, but if it is not set then the runtime gives the message shown below and the allocation fails.

[XRT] ERROR: Host buffer (0) has no bank assignment and is not used as kernel argument before first enqueue operation.

For all other runtimes, this flag is not set, and the runtime copies the input arrays from host memory to device global memory.

5.2.5 Aligned allocations to ensure efficient data transfer. In order to enable aligned transfers from the host to the device global memory, it's necessary that host memory allocations are on 4096 byte boundaries. If not, then the runtime issues a warning message that extra copies will be necessary.

[XRT] WARNING: unaligned host pointer '0x7f3d97c13010' detected, this leads to extra memcpy

To avoid this, a custom allocator needs to be used by std :: vector. std:: vector<site, aligned_allocator $<$ site $>>$

With the strict type checking of $\mathrm{C}++$, this requires that the custom allocator be specified in the function wrapper for the OpenCL implementation of SU3_Bench too. Although this is a minor issue, it does require a \#define to be used around the function call API to distinguish the Xilinx implementation. 
5.2.6 Use HLS instead of OpenCL semantics. Although not investigated in this study, many of the above issues are best addressed by using the Xilinx HLS programming directives instead of OpenCL [15, Chap. 7]. For example with HLS it is recommended that input pointers are declared as type ap_uint $<512>*$ to ensure a 512 bit width transfer. In addition, this would require that the host program pad any input buffers to a 512 bit word size. It is still necessary to use explicit local memory scratch pads and have a copy loop for the compiler to enable burst transfers, by inferring the extent of the data transfer.

It is also possible to set up a data flow pipeline using HLS directives and form a Read $\rightarrow$ Compute $\rightarrow$ Write pipeline where each phase is a separate function. Based on the profiling and timing analysis of the SU3_Bench kernel, this is most likely the best approach for improving performance and can be a topic of future exploration.

\section{RELATED WORK}

Over the last few years, most of the effort applied towards accelerating Lattice QCD codes has been directed at GPUs. However, there have been some recent studies investigating the use of FPGAs as accelerators. Here we summarize those papers that have looked at either the Lattice QCD application domain and/or the usability and portability aspects of the available OpenCL toolchains, or have done similar studies with other HPC kernels on the same devices.

The most recent relevant work can be found in [11] and [10]. Here the authors focus on accelerating the Lattice QCD conjugate gradient algorithm on FPGAs, but use HLS as the language and limit their effort to a single FPGA, with no effort to address portability concerns. In addition, they limit their analysis to the achieved computational rate, and do not investigate performance relative to the capabilities of the device's global memory architecture, and instead estimate performance based on the fact that the input lattice is resident in FPGA local memory, such as device block RAM.

Although not a Lattice QCD kernel, in [6] an OpenCL implementation of a dense matrix-matrix multiplication (SGEMM) kernel on the Arria 10 FPGA was investigated. Interestingly, they obtained better performance with a NDRange kernel vs. a SWI kernel, contrary to the findings of this paper. Although the reason was not investigated, perhaps in this case it is due to the ability to do data blocking to increase reuse, and the computation was long enough to overcome the inherent extra runtime overhead of the NDRange kernel that was observed in our study. A similar study was performed looking at a sparse linear algebra kernel, again investigating both NDRange and SWI kernels [5]. They also found an advantage to using an NDRange kernel for some of the matrices.

The work in [2] examines the optimization of the Nekbone proxy application using HLS, and adopting a data flow kernel design. Although this study is not focused on Lattice QCD, it does provide an excellent account on the level of effort required to optimize an HPC kernel to a FPGA using HLS, in this case also targeting the Xilinx Alveo U280. One outcome of our study is that in order to get further optimizations it is most likely necessary to adopt data flow techniques, and the advanced capabilities of HLS in order to obtain high performance on FPGAs.

\section{CONCLUSIONS}

In this study, we examined porting a memory bandwidth bound microbenchmark written in OpenCL to two different FPGAs, and in particular evaluated the efficacy of the respective OpenCL toolchains. It is not the purpose of this study to compare the two FPGAs head to head, but to evaluate their performance relative to their maximum capabilities. Since the SU3_Bench microbenchmark is inherently memory bandwidth bound, performance efficiency is relative to the maximum performance of their respective memory subsystems. For the Intel Arria 10 FPGA this was a dual-channel DDR based memory subsystem, and for the Xilinx Alveo U280 FPGA we examined its HBM memory subsystem.

Both environments were relatively easy to use and obtain initial results, but in order to obtain relatively good performance, code changes to the OpenCL implementations of the kernel were required.

The first is to covert the kernel to a single work item (SWI) implementation. This is counter to a multi-threaded NDRange kernel necessary for a CPU or GPU implementation. However, this is a relatively easy modification as it just requires the addition of an additional outer loop. It has been demonstrated that a SWI kernel performs more than an order of magnitude better than an equivalent NDRange implementation. It would be highly desirable to make NDRange kernels more efficient, which would ease the task of porting from a multi-threaded NDRange GPU/CPU implementation, and to ease the effort needed to scale to more compute units on the device.

The more significant impact on the code is the lack of a cache architecture in FPGAs and the need to use local block RAM as a scratch pad. This is mitigated by the Intel toolchain as it implicitly creates a cache for each load/store unit it creates, and the size of the cache is configurable. However, the Xilinx toolchain requires that the programmer explicitly declare local memory arrays and create copy loops to move data in and out of global main memory. In addition, the code must be written in a manner that allows the Xilinx compiler to detect the copy operation, and the data structures must be configured such that the compiler inserts 512 bit ( 64 byte) transfers. If it doesn't, then the default is to use 64 bit transfers which results in an $8 x$ loss of bandwidth.

Many of the performance concerns associated with the Xilinx toolchain can be mitigated to some degree by using HLS instead of OpenCL, as HLS allows one to code around many of the limitations we found in this study. However, this is at the expense of portability.

Another portability concern between the two compiler environments is the Intel compiler requires explicit unrolling, and it implicitly does pipelining. This is the opposite of the Xilinx compiler which requires an explicit pragma to pipeline, and implicitly will unroll a loop.

The Xilinx toolchain also requires modifications to the host code, in particular it was necessary to ensure aligned allocations using a custom $\mathrm{C}++$ std :: vector allocator.

In summary, porting a simple Lattice QCD kernel to a FPGA can require a significant restructure of the computational kernel in order to obtain good performance, in particular to account for a lack of a local memory cache generation by the Xilinx toolchain. The final performance achieved with the Intel Arria 10 was $47.6 \%$ of it's 
maximum sustained bandwidth, while for the Xilinx Alveo U280 we achieved $35.2 \%$. Similar GPU based studies have shown $75 \%$ to $90 \%$ efficiencies. In order to improve FPGA efficiencies, future implementations will need to be transformed to a systolic, dataflow solution, which is more natural for a FPGA architecture. In addition, the use of more advanced HLS programming directives may be necessary.

\section{ACKNOWLEDGMENTS}

This research used resources of the National Energy Research Scientific Computing Center (NERSC), a U.S. Department of Energy Office of Science User Facility operated under Contract No. DEAC02-05CH11231.

\section{REFERENCES}

[1] G. Bauer, S. Gottlieb, and T. Hoefler. 2012. Performance Modeling and Comparative Analysis of the MILC Lattice QCD Application su3_rmd. In 2012 12th IEEE/ACM International Symposium on Cluster, Cloud and Grid Computing (ccgrid 2012). 652-659. https://doi.org/10.1109/CCGrid.2012.123

[2] Nick Brown. 2020. Exploring the acceleration of Nekbone on reconfigurable architectures. In Sixth International Workshop on Heterogeneous High-performance Reconfigurable Computing (H2RC'20).

[3] M.A. Clark, R. Babich, K. Barros, R.C. Brower, and C. Rebbi. 2010. Solving lattice QCD systems of equations using mixed precision solvers on GPUs. Computer Physics Communications 181, 9 (Sep 2010), 1517-1528. https://doi.org/10.1016/j. cpc.2010.05.002

[4] Douglas Doerfler, Chris Daley and Thomas Applencourt. 2020. SU3_bench, a Micro-benchmark for Exploring Exascale Era Programming Models, Compilers and Runtimes. https://p3hpcforum2020.alcf.anl.gov/wp-content/uploads/sites/8/ 2020/09/P3HPC_Doerfler_Day-1.pdf. Accessed: 2020-10-29.

[5] Federico Favaro, Ernesto Dufrechou, Pablo Ezzatti, and Juan P. Oliver. 2020 Exploring FPGA Optimizations to Compute Sparse Numerical Linear Algebra Kernels. In Applied Reconfigurable Computing. Architectures, Tools, and Applications, Fernando Rincón, Jesús Barba, Hayden K. H. So, Pedro Diniz, and Julián Caba (Eds.). Springer International Publishing, Cham, 258-268.

[6] Steven Harris, Roger D Chamberlain, and Christopher Gill. 2020. OpenCL Performance on the Intel Heterogeneous Architecture Research Platform. In Proc. of IEEE High-Performance Extreme Computing Conference (HPEC).

[7] Intel Corp. [n.d.]. Intel Arria 10 Device Datasheet. A10-DATASHEET | 2020.06.26.

[8] Intel Corp. [n.d.]. Intel FPGA SDK for OpenCL Pro Edition: Best Practices Guide. UG-OCL003 | 2020.09.28.

[9] Khronos.org. 2012. The OpenCL Specification. Version: 1.2, Document Revision 19, Editor: Aaftab Munshi.

[10] G. Korcyl and P. Korcyl. 2020. Optimized implementation of the conjugate gradient algorithm for FPGA-based platforms using the Dirac-Wilson operator as an example. arXiv:2001.05218 [cs.DC] https://arxiv.org/abs/2001.05218

[11] Grzegorz Korcyl and Piotr Korcyl. 2020. Towards Lattice Quantum Chromodynamics on FPGA devices. Computer Physics Communications 249 (Apr 2020), 107029. https://doi.org/10.1016/j.cpc.2019.107029

[12] NERSC Proxy App Suite. [n.d.]. SU(3) matrix-matrix multiply microbenchmark. https://gitlab.com/NERSC/nersc-proxies/su3_bench/-/tree/fpga Accessed December 1st, 2020.

[13] T. Nguyen, S. Williams, M. Siracusa, C. MacClean, D. Doerfler, and N. J. Wright. 2020. The Performance and Energy Efficiency Potential of FPGAs in Scientific Computing. In 2020 IEEE/ACM Performance Modeling, Benchmarking and Simulation of High Performance Computer Systems (PMBS). 8-19. https: //doi.org/DOI10.1109/PMBS51919.2020.00007

[14] Xilinx Inc. [n.d.]. Alveo U280 Data Center Accelerator Card Data Sheet. DS963 (v1.3) May 11, 2020.

[15] Xilinx Inc. [n.d.]. Vitis Unified Software Platform Documentation, Application Acceleration Development. UG1393 (v2019.2) February 28, 2020.

\section{A KERNEL SOURCE CODE LISTINGS}

This appendix provides listings to aid in understanding the main text and analysis. All source code used in this study can be accessed at the URL found in [12].

Numerous versions of the su3_bench kernel were developed in the course of this study. The following table provides a cross reference between the kernel name in the online repository and the listing reference in this paper. The Arria 10 kernels can be found in the directory Intel, and Xilinx kernels can be found in the directory Xilinx/src.

Table 7: Listing to Kernel Name Cross Reference

\begin{tabular}{|l|c|c|c|}
\hline Listing & Type & Arria 10 & Xilinx U280 \\
\hline 2 & NDRange & k_mat_nn3b.cl & \\
\hline 3 & NDRange & & k_mat_nn6.cl \\
\hline 4 & SWI & & k_mat_nn30a.cl \\
\hline 5 & SWI & k_mat_nn10.cl & \\
\hline 6 & SWI & & k_mat_nn32.cl \\
\hline 7 & SWI & & k_mat_nn33.cl \\
\hline
\end{tabular}

\section{SU(3) Matrix Structure}

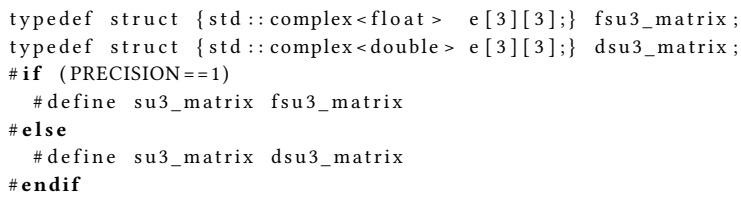

\section{Site Structure}

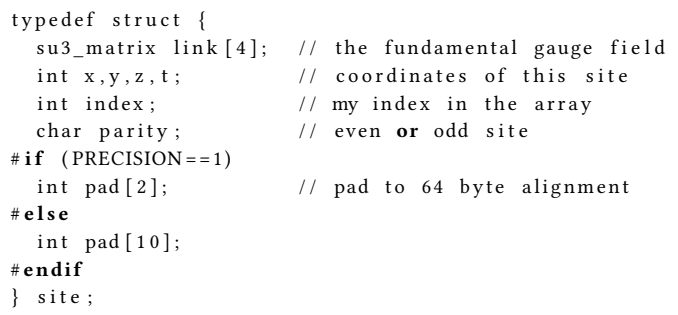

\section{Complex Multiply/Sum Macro used by the Kernels}

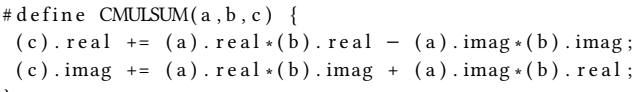

\section{Listings}

\section{Listing 1: NDRange Kernel}

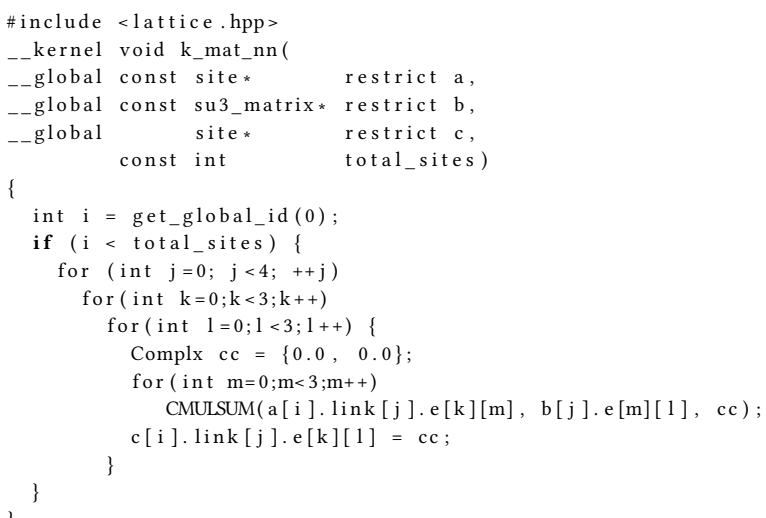




\section{Listing 2: NDRange Kernel with Unroll}

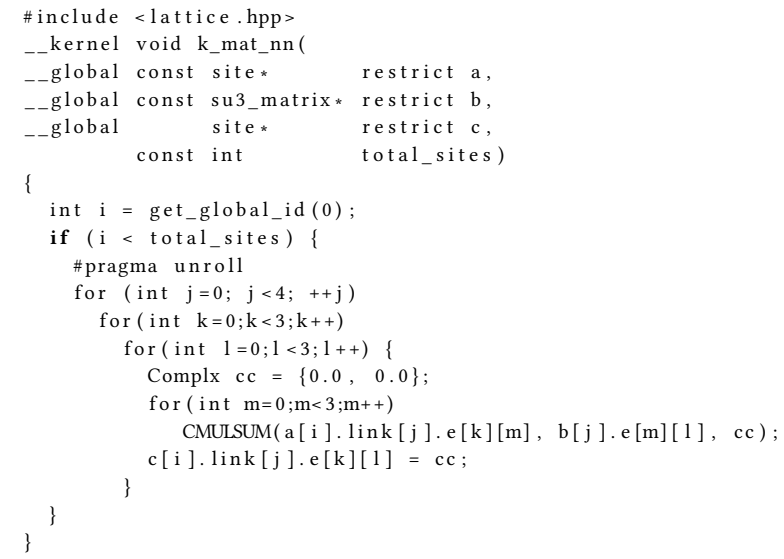

\section{Listing 3: NDRange Kernel with 16 workers}

\#include <lattice.hpp>

\#define WG_SIZE 16

_-_attribute_-_ ( reqd_work_group_size (WG_SIZE, 1, 1) )

_- kernel void k_mat_nn(

_- global const site * restrict a,

global const su3_matrix* restrict b.

_- global site* restrict c, const int total_sites)

int $\mathrm{i}=$ get_global_id $(0)$;

int $i \mathrm{i}=$ get_local_id $(0)$;

if ( $i>$ total_sites)

return;

site a_buf [WG_SIZE]

site c_buf[WG_SIZE];

su3_matrix b_buf [4];

if $(\mathrm{i} i<4)$

b_buf [ii $]=b[$ ii $]$;

barrier (CLK_LOCAL_MEM_FENCE)

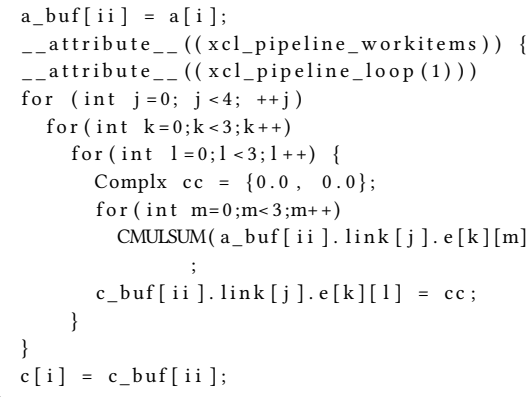

\section{Listing 4: SWI Kernel}

\#include <lattice.hpp>

_-kernel void k_mat_nn(

global const site *

restrict a,

_ global const su3_matrix* restrict b,

-_global site* restrict c,

\{

$$
\text { const int total_sites) }
$$

for (int $i=0 ; i<$ total_sites; ++i) \{

for (int $\mathrm{j}=0 ; \mathrm{j}<4 ;++\mathrm{j}$ )

for (int $\mathrm{k}=0 ; \mathrm{k}<3 ; \mathrm{k}++$ )

for (int $1=0 ; 1<3 ; 1++$ ) \{

Complx cc $=\{0.0,0.0\}$

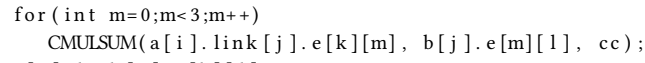

\section{Listing 5: SWI Kernel with Unroll}

\#include <lattice.hpp>

kernel void k mat nn

-global const site* restrict a,

_-_global const su3_matrix* restrict b,

_-_global site* restrict c,

const int total_sites )

for (int $\mathrm{i}=0 ;$ i<total_sites; ++i)

\# pragma unroll

for $($ int $\mathrm{j}=0 ; \mathrm{j}<4 ;++\mathrm{j})$

for ( int $\mathrm{k}=0 ; \mathrm{k}<3 ; \mathrm{k}++$ )

for (int $1=0 ; 1<3 ; 1++) \quad\{$

Complx cc $=\{0.0,0.0\}$;

for (int $\mathrm{m}=0 ; \mathrm{m}<3 ; \mathrm{m}++$ )

$\operatorname{CMULSUM}(\mathrm{a}[\mathrm{i}]$. link $[\mathrm{j}] . \mathrm{e}[\mathrm{k}][\mathrm{m}], \mathrm{b}[\mathrm{j}] . \mathrm{e}[\mathrm{m}][1], \mathrm{cc})$; $c[i] \cdot \operatorname{link}[j] . e[k][1]=c c$;

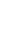

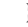

\section{Listing 6: SWI Kernel using a Scratch Pad}

\#include <lattice.hpp

\#define BUF SIZE

kernel__attribute_-_ ((reqd_work_group_size $(1,1,1)))$

_-_kernel void k_mat_nn(

_- global const site*

matrix* restrict b,

_-_global site* restrict c, const int total_sites

site a_buf[BUF_SIZE];

site c_buf[BUF_SIZE];

su3_matrix b buf[4];

for (int $j=0 ; j<<, ;++j$ )

for (int $\mathrm{k}=0 ; \mathrm{k}<3 ;++\mathrm{k}$ )

for (int $1=0 ; \quad 1<3 ;++1$ )

b_buf $[j] . e[k][1]=b[j] \cdot e[k][1]$;

for (int $i=0 ; \quad i<$ total_sites; $i \quad+=$ BUF_SIZE)

int chunk size = BUF_SIZE ;

if $((i+$ BUF_SIZE $)>$ total_sites $)$

chunk_size $=$ total_sites $-i$;

_- attribute__( $\left(\left(x c l_{-}\right.\right.$pipeline_loop (1) ) )

for (int ii $=0 ;$ ii $<$ chunk_size; ++ii) \{

for (int $\mathrm{j}=0 ; \mathrm{j}<4 ;++\mathrm{j}$ )

for $($ int $\mathrm{k}=0 ; \mathrm{k}<3 ;++\mathrm{k})$

for (int $1=0 ; 1<3 ;++1)$

a_buf[ii $] \cdot \operatorname{link}[j] . e[k][1]=a[i+i i] \cdot \operatorname{link}[j] \cdot e[k][1]$;

for (int $\mathrm{j}=0 ; \mathrm{j}<4 ;++\mathrm{j})$

for (int $\mathrm{k}=0 ; \mathrm{k}<3 ; \mathrm{k}++$ )

for (int $1=0 ; 1<3 ; 1++)$

Complx cc $=\{0.0,0.0\}$

for (int $\mathrm{m}=0 ; \mathrm{m}<3 ; \mathrm{m}++$ )

$\operatorname{CMULUUM}\left(a_{-} b u f[\right.$ ii $]$. link $[\mathrm{j}] . e[k][\mathrm{m}]$, b_buf $[\mathrm{j}]$.e $[\mathrm{m}][$ ]$, \mathrm{cc})$;

c_buf[ii $]$.link $[j] . e[k][1]=c c$;

for ( int $\mathrm{j}=0 ; \mathrm{j}<4 ;++\mathrm{j}$ )

for (int $\mathrm{k}=0 ; \mathrm{k}<3 ;++\mathrm{k}$ )

for (int $1=0 ; 1<3 ;++1)$

$c[i+i i] \cdot \operatorname{link}[j] \cdot e[k][1]=c_{-} b u f[i i] \cdot \operatorname{link}[j] \cdot e[k][1]$;

\} 


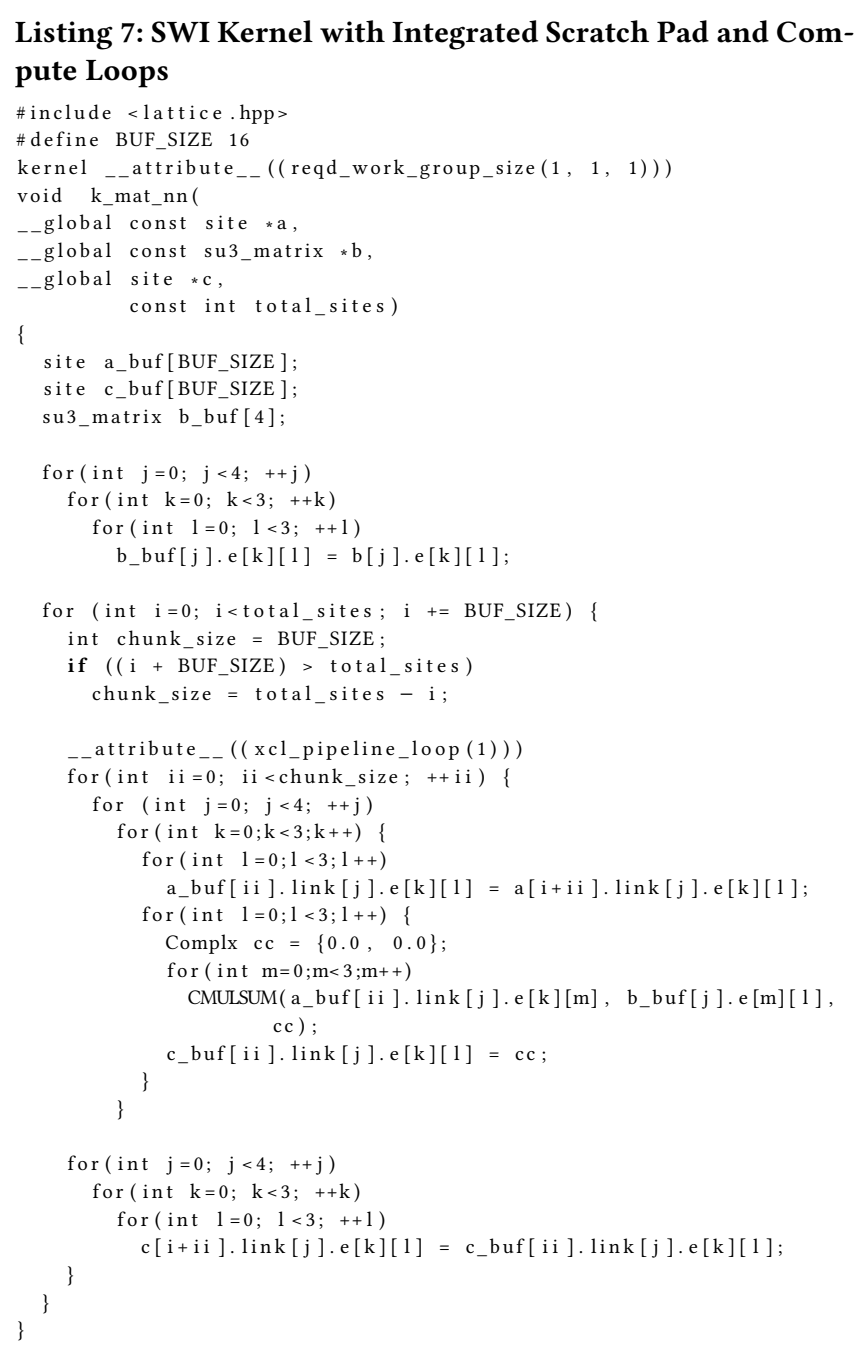

